\title{
Light and shadow in the Galactic Center
}

On the detection of the relativistic periastron shift of star S2 in the Galactic Center

\author{
Andreas Eckart $^{1,2 *}$, M. Parsa ${ }^{1,2}$, E. Mossoux ${ }^{3}$ B. Shahzamanian ${ }^{1,4}$, M. Zajacek $^{1,2}$, E. \\ Hosseini $^{1,2}$, M. Subroweit ${ }^{1}$, F. Peissker ${ }^{1}$, N. Sabha ${ }^{1}$, M. Valencia-S. ${ }^{1}$, C. \\ Straubmeier $^{1}$, V. Karas ${ }^{5}$, S. Britzen ${ }^{2}$, A. Zensus ${ }^{2}$ \\ 1) I. Physikalisches Institut der Universität zu Köln, Zülpicher Str. 77, D-50937 Köln, Germany; \\ 2) Max-Planck-Institut für Radioastronomie, Auf dem Hügel 69, D-53121 Bonn, Germany; \\ 3)Space sciences, Technologies and Astrophysics Research (STAR) Institute, Université de Liège, \\ Allée du 6 Août, 19c, Bât B5c, 4000 Liège, Belgium; \\ 4) Instituto de Astrofisica de Andalucia (CSIC), Glorieta de la Astronomia s/n, 18008 Granada, \\ Spain \\ 5) Astronomical Institute of the Academy of Sciences Prague, Bocni II 1401/1a, CZ-141 31 \\ Praha 4, Czech Republic \\ E-mail: eckarteph1.uni-koeln.de
}

\begin{abstract}
We report on the nature of prominent sources of light and shadow in the Galactic Center. With respect to the Bremsstrahlung X-ray emission of the hot plasma in that region the Galactic Center casts a 'shadow'. The 'shadow' is caused by the Circum Nuclear Disk that surrounds SgrA* at a distance of about 1 to 2 parsec. This detection allows us to do a detailed investigation of the physical properties of the surroundings of the super massive black hole. Further in, the cluster of high velocity stars orbiting the central super massive black hole SgrA* represents an ideal probe for the gravitational potential and the degree of relativity that one can attribute to this area. Recently, three of the closest stars (S2, S38, and S55/S0-102) have been used to conduct these investigations. In addition to the black hole mass and distance a relativistic parameter defined as $=r_{s} / r_{p}$ could be derived for star S2. The quantity $r_{s}$ is the Schwarzschild radius and $r_{p}$ is the pericenter distance of the orbiting star. Here, in this publication, we highlight the robustness and significance of this result. If one aims at investigating stronger relativistic effects one needs to get closer to SgrA*. Here, one can use the emission of plasma blobs that orbit SgrA*. This information can be obtained by modeling lightcurves of bright X-ray flares. Finally, we comment on the shadow of the SgrA* black hole expected due to light bending and boosting in its vicinity.
\end{abstract}

Frontier Research in Astrophysics - III

28 May - 2 June, 2018

Palermo, Italy

\footnotetext{
* Speaker.
} 


\section{Introduction}

The Galactic Center is a very active region. It is filled with stars, dust, gas at different temperatures and different ionization states, and an accreting super massive black hole (SMBH) at the very center of the region, i.e. Sagittarius A* (SgrA*, for an overview see, e.g., Eckart et al. 2017). Due to the large mass of SgrA*, it amounts to about 4 million solar masses, the motion and radiation is also subjected to relativistic effects, which can be used to map out space time in the vicinity of SgrA*. This leads to a situation in which many competitive sources of radiation and extinction or dilution act at the same time. Hence, the region can be thought of as an interplay between light and shadow. In this brief overview, we begin the discussion at a scale of several parsecs and investigate the X-ray Bremsstrahlung radiation of the plasma, in which SgrA* and the central stellar cluster is embedded. Further in we detect the relativistic motion of the luminous star S2. Martins et al. (2008) confirmed that the star S2 is a main-sequence star of spectral type B0-2.5 V with a zero-age main-sequence (ZAMS) mass of $19.5 \mathrm{M}_{\odot}$. Getting close to the last stable orbit around the SMBH we have indications for plasma moving at relativistic speed and finally there may be the possibility to see the shadow of the SMBH as it bends the light generated in its accretion zone.

\subsection{Shadow of the Circum Nuclear Disk}

The SgrA complex at the center of the Milky Way consists of several components like the SMBH SgrA*, the mini-spiral, and the Circum Nuclear Disk (CND). The source complex can be observed in the radio/sub-millimeter, infrared and X-ray wavelength domain. However, until now and as a part of SgrA East, the CND had only been detected in the radio and far infrared. Thanks to the 4.6 Ms of Chandra observations of the Galactic Center region, as described by Mossoux \& Eckart (2018), we were now able to detect an X-ray "shadow" of the CND against the diffuse X-ray emission of the entire region. Mossoux \& Eckart (2018) aimed at finding out if the CND acts as an absorber for the diffuse X-ray emission or as a barrier for the plasma close to Sgr A* and the central stellar cluster. The basis for this investigation are the ACIS-I and ACIS-S/HETG Chandra data covering the time interval between 1999 and 2012. Subtracting a smooth model of the diffuse $\mathrm{X}$-ray emission from the image, inverting it, clipping it at zero intensity, and smoothing the result gives an image of the CND with identifiable subcomponents. The result is rather insensitive to the diffuse X-ray emission model.

The shadow inspired us to define several regions (inside, on, and outside the CND in addition to regions off the entire structure) for which we extracted spectra. From the spectra we derived temperatures and column densities. The general picture Mossoux \& Eckart (2018) get is that best fits to the spectra are obtained assuming a two temperature plasma. For the CND a single temperature model with $1.8 \mathrm{keV}$ and total local column density of $2.3 \times 10^{22} \mathrm{~cm}^{-2}$ of the hot gas is obtained. Inside the CND temperatures of $1 \mathrm{keV}$ and $5 \mathrm{keV}$ are needed. Similarly for the outside temperatures of $0.35 \mathrm{keV}$ and $1.3 \mathrm{keV}$ are indicated. Details of the MCMC fitting results are given in Table 1 by Mossoux \& Eckart (2018). We find that the plasma at the location of the CND is cooler in general and may in fact act as a barrier between the hot plasma insight and outside of the CND. 


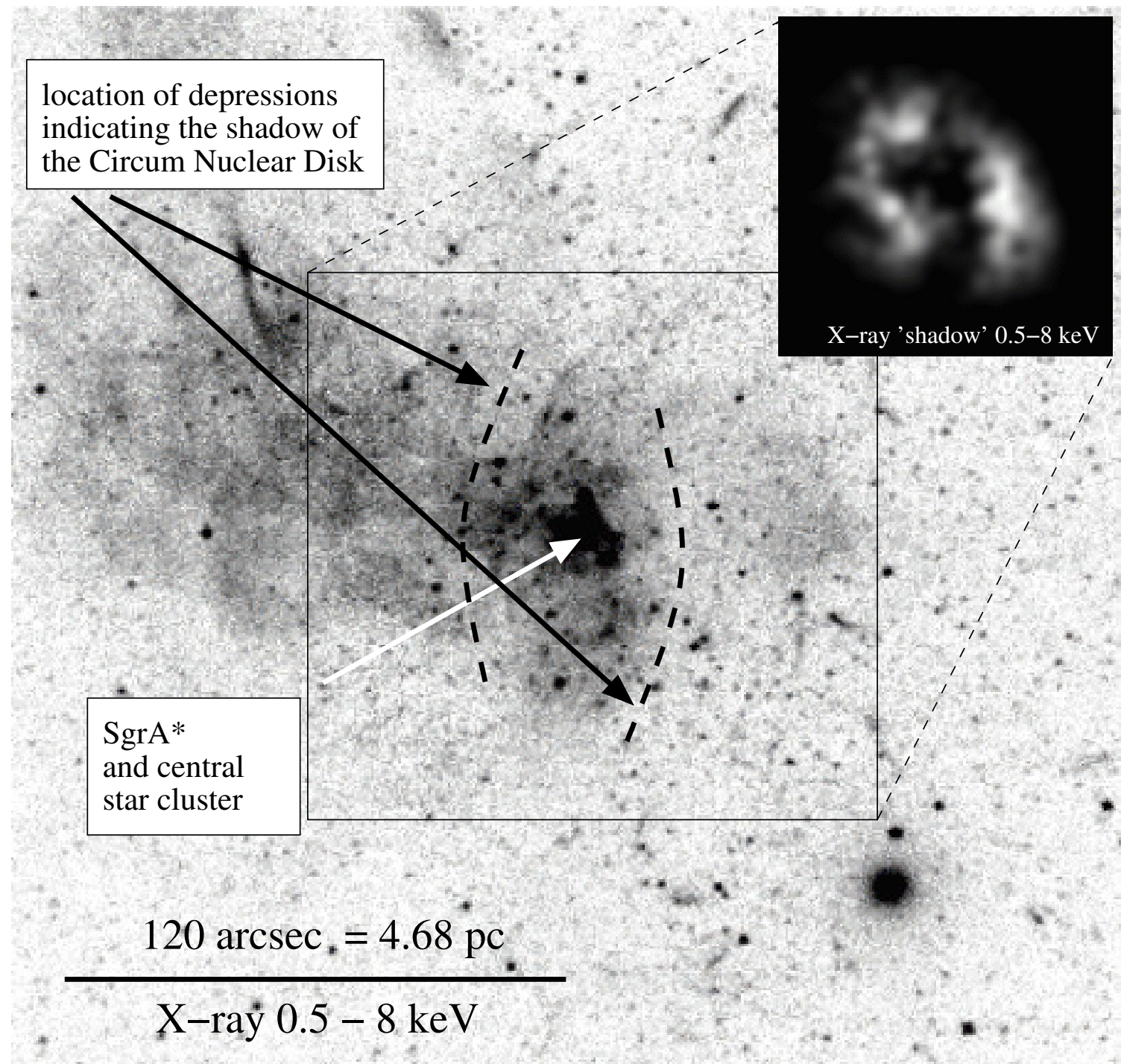

Figure 1: The Chandra image of the X-ray diffuse emission at the Galactic Center as shown by Mossoux \& Eckart (2018) and as obtained for the energy interval between 0.5 and $8 \mathrm{keV}$. The observations have been carried out between 1999 and 2012. Corresponding to the Chandra angular resolution the pixel size is half an arcsecond. The image intensity represents the count rate. It is shown in an inverted logarithmic scale implying that the brightest areas are represented as the darkest regions. The location of SgrA* and the central stellar cluster is shown. The dashed line shows depressions due to the shadowing effect by the CND. In Mossoux \& Eckart (2018) we explain the algorithm that allowed us to transform these indications of the shadow into an image of it. The inset shows the image of the shadow which looks very similar to the ALMA image of the CND. Its inner edge is identical to the circumference of the bright part of the central stellar cluster (see figures in Mossoux \& Eckart 2018). 


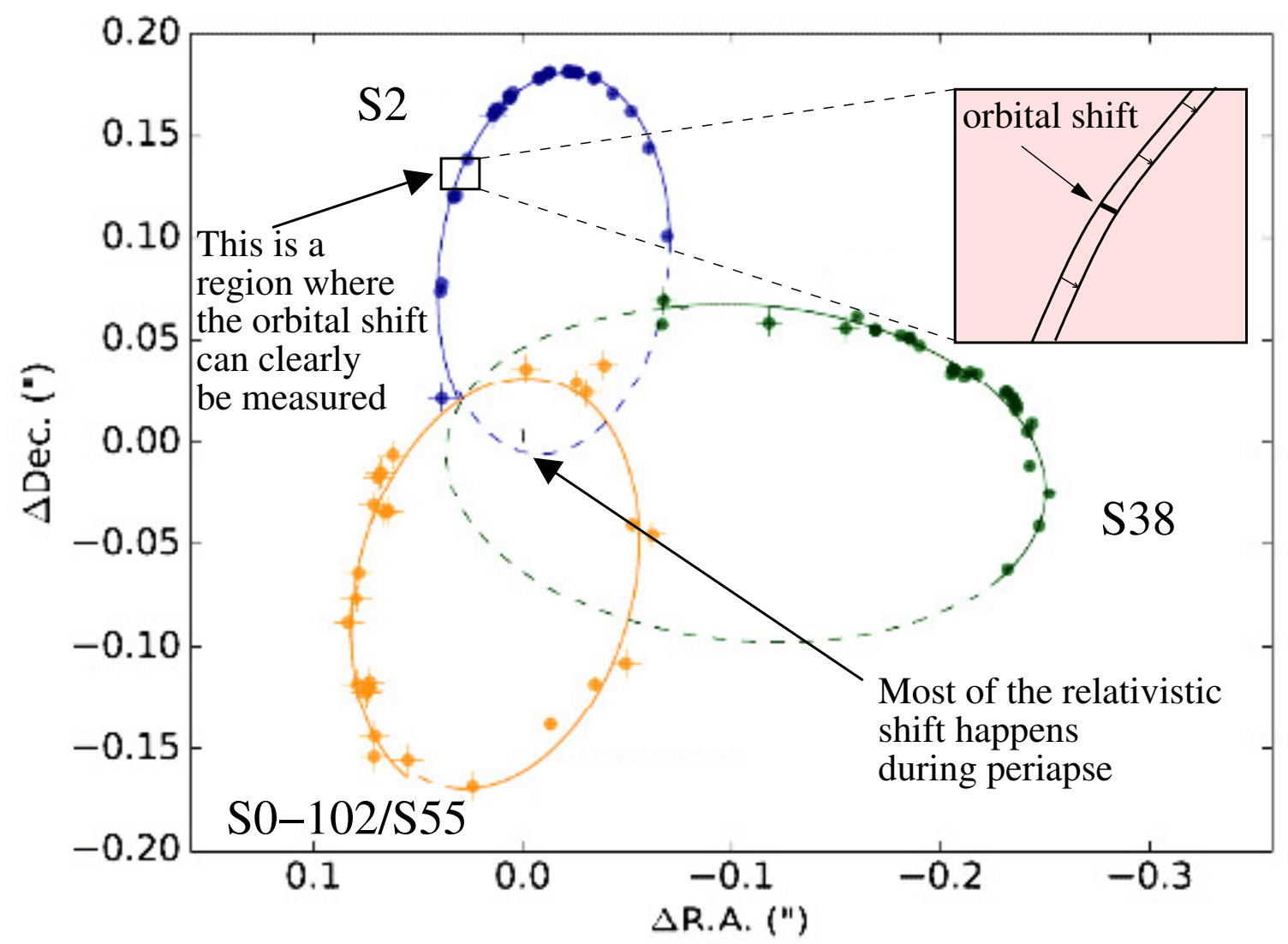

Figure 2: Orbital fits for the high velocity stars S2, S38, and S0-103/S55 orbiting the Galactic Center SMBH SgrA* as taken from Parsa et al. (2017). We indicated regions in which the relativistic periastron shift is imprinted onto the orbit and where the effects can be seen easily in comparison to the larger scale orbit. The zoom depicts the (projected) prograde advance of the orbit by the amount $\Delta \omega$. See also text and animation in the ESO press Announcemant ann17051 (http://www.eso.org/public/announcements/ann17051/).

\section{Relativistic motion of star S2}

The S-star cluster that surrounds the supermassive black hole SgrA* in the Galactic center is ideally suited to investigate the physics close to this peculiar object. In particular such a study allows us to perform dynamical tests of general relativity. Using positions and radial velocities of three high velocity stars with the shortest periods (S2, S38, and S55/S0-102; see Fig.2) we derived in Parsa et al. (2017) a black hole mass of $M_{B H}=(4.15 \pm 0.13 \pm 0.57) \times 10^{6} \mathrm{M}_{\odot}$ and a distance to it of $R_{0}=8.19 \pm 0.11 \pm 0.34 \mathrm{kpc}$. The possibility of the detection of faint stars inside the S2 orbit is discussed in Zajacek \& Tursunov (2018). The relativistic character of simulated orbits was investigated via a first-order post-Newtonian approximation to calculate stellar orbits that cover a large range of periapse distances. These calculations were used to derive changes in orbital elements that were obtained from fits to different sections of the orbits. Here we used changes in the eccentricity and the semi-major axis obtained from fits to the lower and upper part of the orbits, these are $\Delta e_{l} / \Delta e_{u}$ and $\Delta a_{l} / \Delta a_{u}$. We also calculated the periastron shift $\Delta \omega$ between the pre- and post-periapse part of the orbit. Parsa et al. (2017) could show that these quantities are correlated 
with the relativistic parameter we defined as $=r_{s} / r_{p}$. Here, $r_{s}$ is the Schwarzschild radius and $r_{p}$ is the pericenter distance of the orbiting star. After establishing these correlations we could use the corresponding data from star S2 to derive its relativistic parameter and, hence, determine the degree of relativity that is shown by its orbit. For S2 Parsa et al. (2017) were able to derive a value of $=0.00088 \pm 0.00080$. This value is consistent with the expected value of $=0.00065$ derived for the star S2 using the SgrA* black hole mass. Parsa et al. (2017) argue that this derived value is most likely not dominated by possible perturbing influences such as noise on the derived stellar positions, rotation of the field that was imaged at different epochs, and possible drifts of the black hole in position.
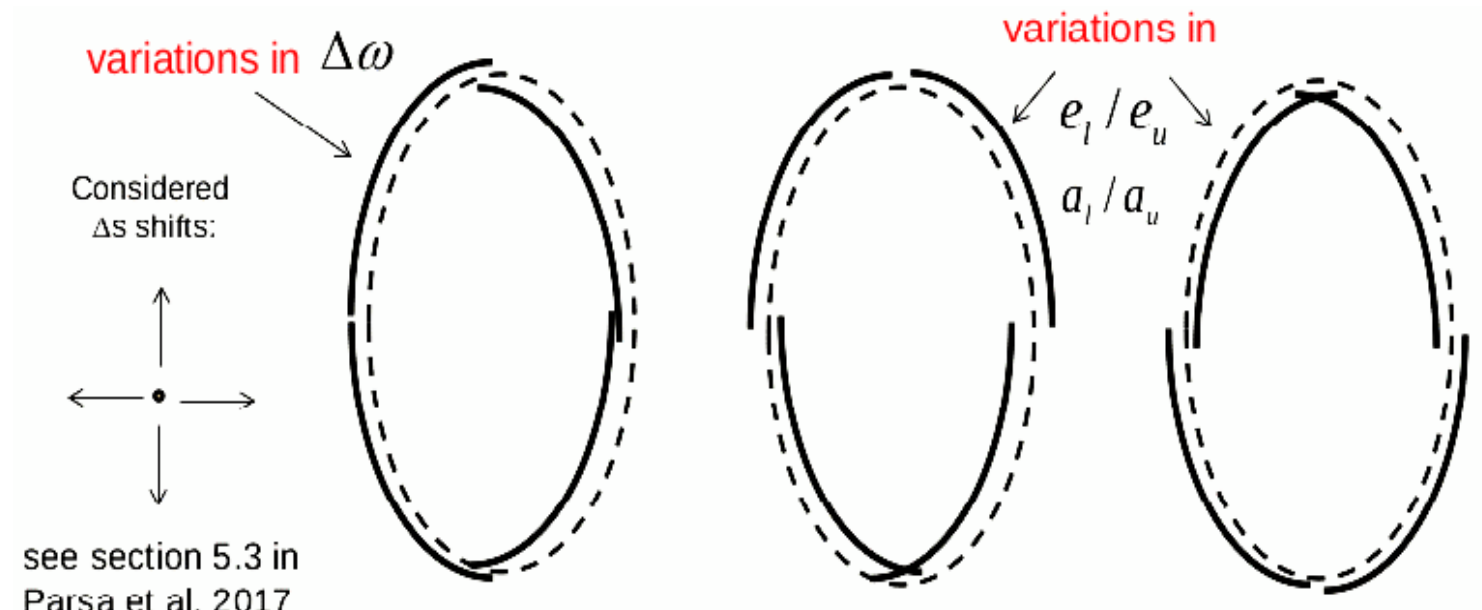

Figure 3: Sketch depicting the randomization of the shifts for the four orbital sections by $\Delta s$. As an example of effective variations in the periastron shift $\Delta \omega$ and the ratios $\Delta e_{l} / \Delta e_{u}, \Delta a_{l} / \Delta a_{u}$ between lower and upper orbital fits are shown. Orbital fits to these configurations were used to obtain the distributions for the assumption of a fully noise dominated case.

In Parsa et al. (2017) we present a qualitative analysis of the robustness and significance of the results in chapter 5.3. Here, we present the results of a numerical calculation of the significance in comparison to a fully noise dominated scenario. The uncertainties for the $e^{-}$, and $a$-ratios as well as the $\Delta \omega$ value were obtained by transporting the uncertainties from the measurements, via the reference frames to the final statement. As we used only images in which $\mathrm{SgrA}^{*}$ could be detected as well, for the stars in the central arcsecond the positional uncertainties are the most important quantities in order to measure the deviation from a Newtonian orbit. We assume a noise dominated case and estimate the uncertainties relative to it. We use the combination of our uncertainty in R.A. direction (essential in the measurement of the $\Delta \omega$ of the $\mathrm{S} 2$ orbit) and the data from the literature. We then find a mean uncertainty of 1.4 mas for an individual position. Knowing that we (Parsa et al. 2017) have about 5 data points per quarter of the three dimensional orbit we derive a positioning uncertainty for each quarter of about $\Delta s=0.5$ mas (in the projected view of the orbit). Hence, we randomize the position of each orbital segment by placing it at $\Delta s=0,+0.5,-0.5$ mas (see Fig.3) with respect to the nominal position in the deprojected Newtonian orbit, i.e., less than that in the projected view of the orbit. For each orbit that we generate with this procedure we calculate the quantities $\Delta e_{u} / \Delta e_{l}, \Delta a_{u} / \Delta a_{l}$, and the periastron shift $\Delta \omega$. The corresponding histograms are shown 
in Figs. 4 and 5. These diagrams can now be used to determine an uncertainty $\sigma$ of the distributions and compare those to the values measured off for star S2. The $e$ - and $a$-ratios and $\Delta \omega$ obtained for

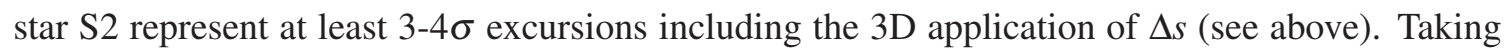
the inclination of $S 2$ of about $137^{\circ}$ into account the 3-4 $\sigma$ excursions turn into a $4-6 \sigma$ result. Parsa et al. (2017) shows that the inclusion of a possible small proper motion of SgrA* with respect to the stellar cluster does not change the result on the relativistic periastron shift (see in particular Tab.8 in Parsa et al. 2017). Hence, it also does not effect our evaluation of the result with respect to the noise dominated Newtonian case.

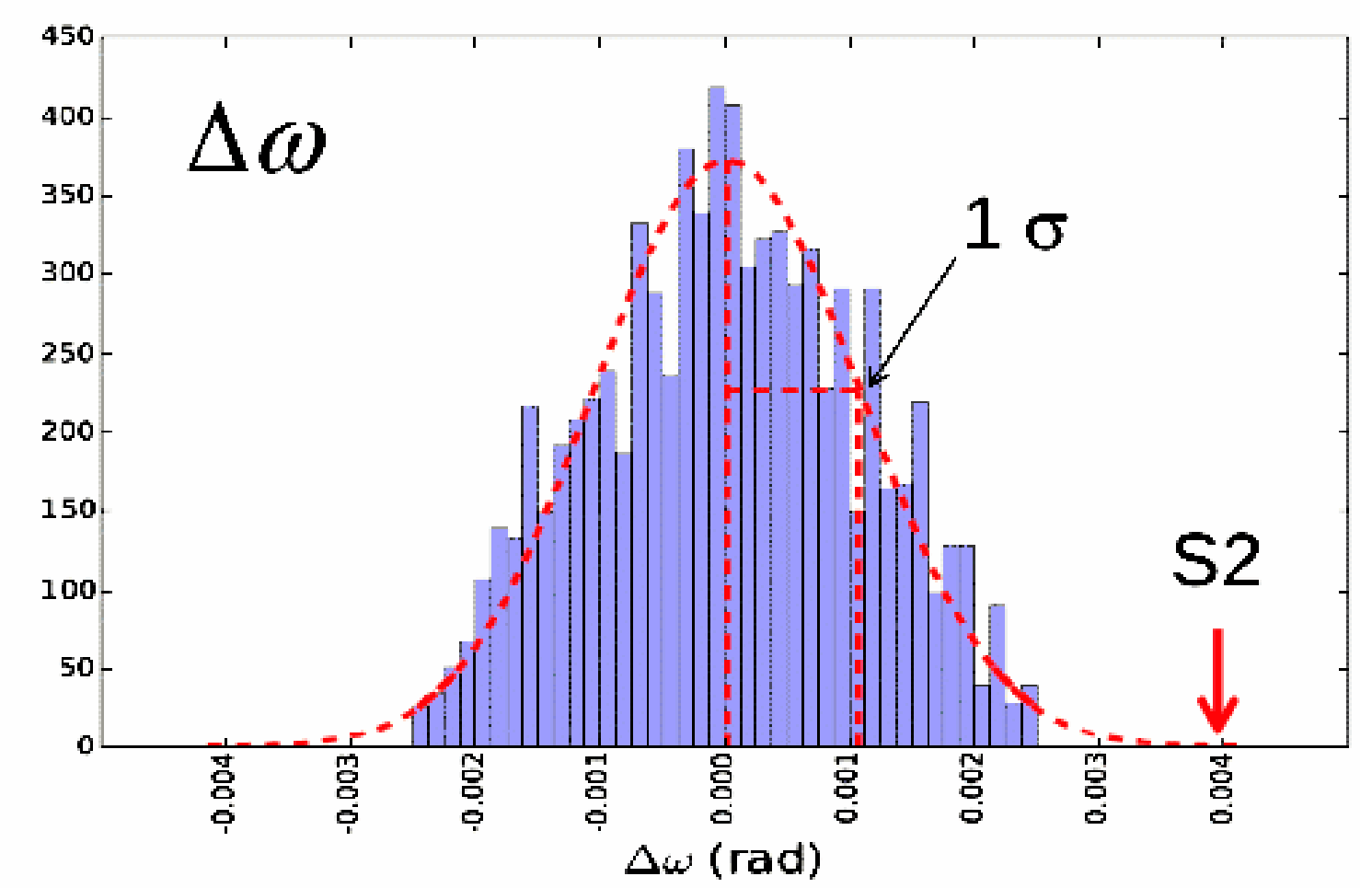

Figure 4: Distribution of the periastron shift $\Delta \omega$ obtained from the orbits with randomization of the shifts for the four orbital sections by $\Delta s$ under the assumption of a fully noise dominated Newtonian case.

\subsection{Relativistic motion of plasma blobs}

Karssen et al. (2017) could show that the lightcurves of bright X-ray SgrA* flares have the exact shape one expects from plasma blobs orbiting the SMBH close to the last stable orbit. This is true for the four bright flares discussed by Karssen et al. (2017) and the recent bright flare reported by Ponti et al. (2017) (see also Eckart et al. 2018). Li, Yuan \& Wang (2017) elaborate on the Li et al. (2015) scenario that explains the SgrA* flares in a magneto-hydrodynamic model. analogous with the theory of solar flares. This involved magnetic field loops originating in the accretion flow and leading via reconnection of the field lines to the well known coronal mass ejection described in solar physics. These bright ejections close to the the accretion flow or disk (see Fig.1 in Li, Yuan \& Wang (2017) may be an explanation for the orbiting blobs that explain the observed X-ray flare emission so well. Recently, the orbiting spot model has obtained compelling observational support 

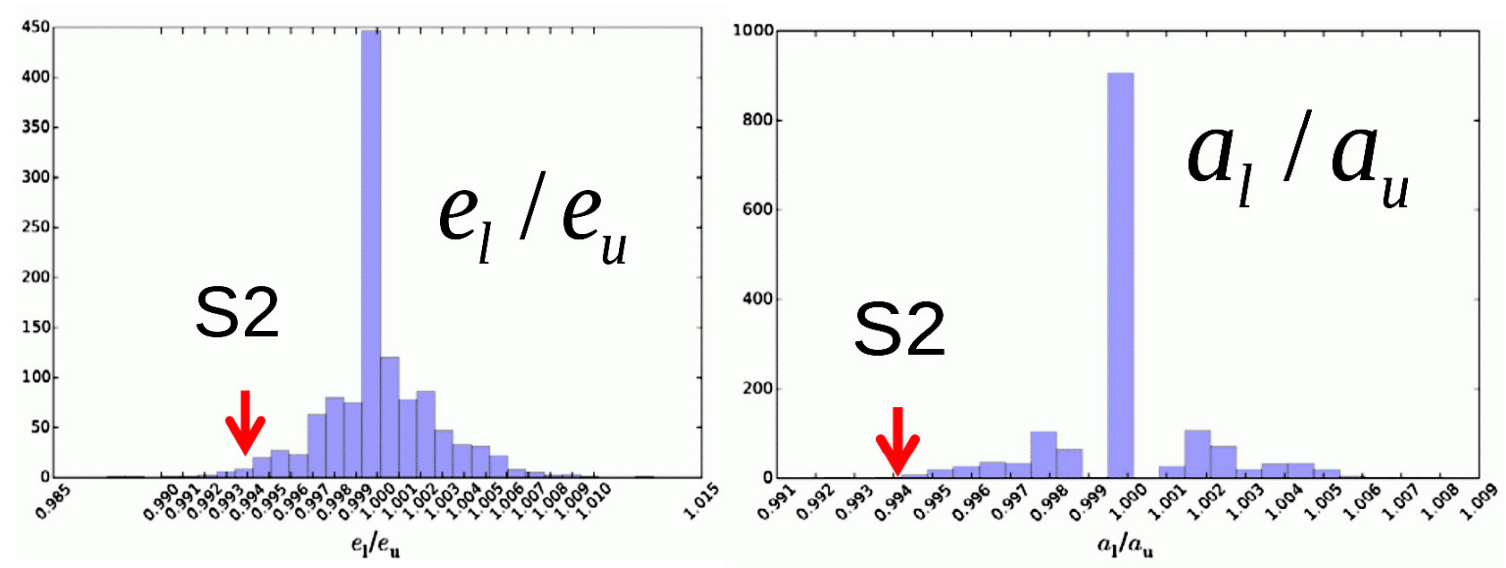

Figure 5: Distribution of the ratios $\Delta e_{l} / \Delta e_{u}, \Delta a_{l} / \Delta a_{u}$ between lower and upper orbital fits obtained from the orbits with randomization of the shifts for the four orbital sections by $\Delta s$ under the assumption of a fully noise dominated Newtonian case.

through the infrared interferometric detection of orbital motions of a dominant emission component near the last stable circular orbit of the massive black hole SgrA* (Gravity Collaboration 2018) and the submillimeter interferometric imaging of a ring of emitting blobs close to the last stable orbit around the super massive black hole in M87 (Event Horizon Telescope Collaboration; 2019a and 2019b).

Since the bright X-ray flares are correlated with synchronous NIR flares (see e.g. Eckart et al. 2012) the finding by Karssen et al. (2017) probably also holds for the NIR domain. However, in the X-ray domain, flares occur at a low rate than in the NIR. Therefore, it is less likely that faint X-ray flares overlap the bright flare events resulting in a clearer agreement with lightcurves expected from orbiting blobs. In the NIR flares are much more frequent and it is less likely to obtain a clean single blob light curve undisturbed by secondary flare events. The assumption that goes in is that the blobs stay stable for a substantial fraction of a single orbit. The characteristics of these theoretical light curves is that they all show a shoulder due to lensing amplification on the ascending part of the boosting dominated light curve (The boosting sets in before the lensing occurs). This can be found in the observed lighcurves of all bright X-ray flares as well. Reassuring is also that by fitting the flare curves scale free (in gravitational time scales $G M / c^{3}$ ) with the theoretical light curves and then scaling them by introducing the observed flare time in seconds one obtains the mass of SgrA*. This can be understood by the fact that the black hole mass scales linearly with the black hole (or rather the event horizon) size,i.e., with the orbital time scale and, therefore, the flare time scale in our model. Hence, being able to explain the X-ray flare shapes via the assumption of orbiting matter is another indication for the high degree of relativity in the vicinity of SgrA*.

\subsection{Shadow of the supermassive black hole}

One of the goals of the Event Horizon Telescope project (EHT) is to get a high angular resolution image at mm-wavelengths of SgrA* in order to measure the so called shadow of the SMBH. This shadow results from the following effect: Luminous plasma is orbiting the SMBH. Due to the bending of light by the SMBH there is the possibility that in addition to a bright Doppler boosted 
part of the source there may appear a dark region in the overall structure (see Falcke et al. 2000). The expected size of that region is of the order of 30-50 $\mu$ as. However, if one is able to see it or not will critically depend on the actual observational and geometrical situation (The shadow is also not a feature uniquely associated to Black holes only - see detailed discussion in Eckart et al. 2017). In order to see this shadow one needs to have a special orientation of the system, the accretion must be well ordered and not chaotic, and it would be best if there is no jet that may disturb the impression one gets from the orbiting material. Another problem may also result from the fact that the scattering screen along the line of sight just becomes transparent around a wavelength of $1 \mathrm{~mm}$. Hence, it may be that multiple simultaneous images (like the speckle effect known from observations at optical and infrared wavelengths) may occur. All of these effects and influences will make it difficult to get a clear view of the immediate environment of SgrA*.

The first EHT results were recently published by Ru-Sen Lu et al. (2018). The authors report on a detection of intrinsic source structure at about 3 Schwarzschild radii, i.e., about $30 \mu$ as and therefore on the expected size scale for the shadow of the black hole. Their Fig. 5 shows possible image structures that one would expect from based on the current amplitude and closure phase measurements. The variations in the data and the appearance of the models are also consistent with the expectation of a shadow, however, detailed images that would show that disk structure due to orbiting matter with indications of a shadow have not yet been published.

\section{Summary and Conclusion}

Luminous stars and plasma blobs can be used to map the space time close to SgrA*. While the presence of the plasma blobs close to the last stable orbit needs to be inferred from the shape of the light curves the stars can be observed directly. The plasma blobs would be part of the structure surrounding the SMBH. Due to light bending effects that structure is potentially showing a shadow of the black hole.

With Parsa et al. (2017) we used three stars to derive the mass and distance of SgrA* in a Newtonian and post-Newtonian solution. A new and simple method that compares properties of Newtonian fits to the lower, upper, pre-, and post-periapse parts of the orbits allows us to determine the degree of relativity. For the high velocity star S2 the values for the $e-$ and $a-$ ratios as well as $\Delta \omega$ value lie close to the values expected for S2 and the SgrA* mass (see Iorio 2017). Here we give an analysis of the uncertainties for these quantities with respect to a noise dominated Newtonian situation. In this case the deviations are significant and the $\mathrm{S} 2$ values for the e- and a-ratios and $\Delta \omega$ represent a $\geq 4 \sigma$ result, and it appears to be highly unlikely that the common effect seen in all three quantities originates from a purely noise dominated scenario. Accepting this result, S2 is the first star with a resolvable orbit around a SMBH for which a test for relativity can be performed. In the near future these results will become more significant using interferometric data as obtained by GRAVITY at the VLTI (e.g., Gravity Collaboration 2017, Mérand et al. 2017).

In addition we report on the 'shadow' the Galactic Center casts with respect to the X-ray Bremsstrahlung emission of the plasma in the local for- and background. The 'shadow' is caused by The Circum Nuclear Disk that surrounds SgrA* casts a 'shadow' that allows us to effectively subdivide the hot nuclear plasma into areas inside, at, and outside the CND. Two temperature models and estimates of the column density give us a clear picture of the physical conditions of the 


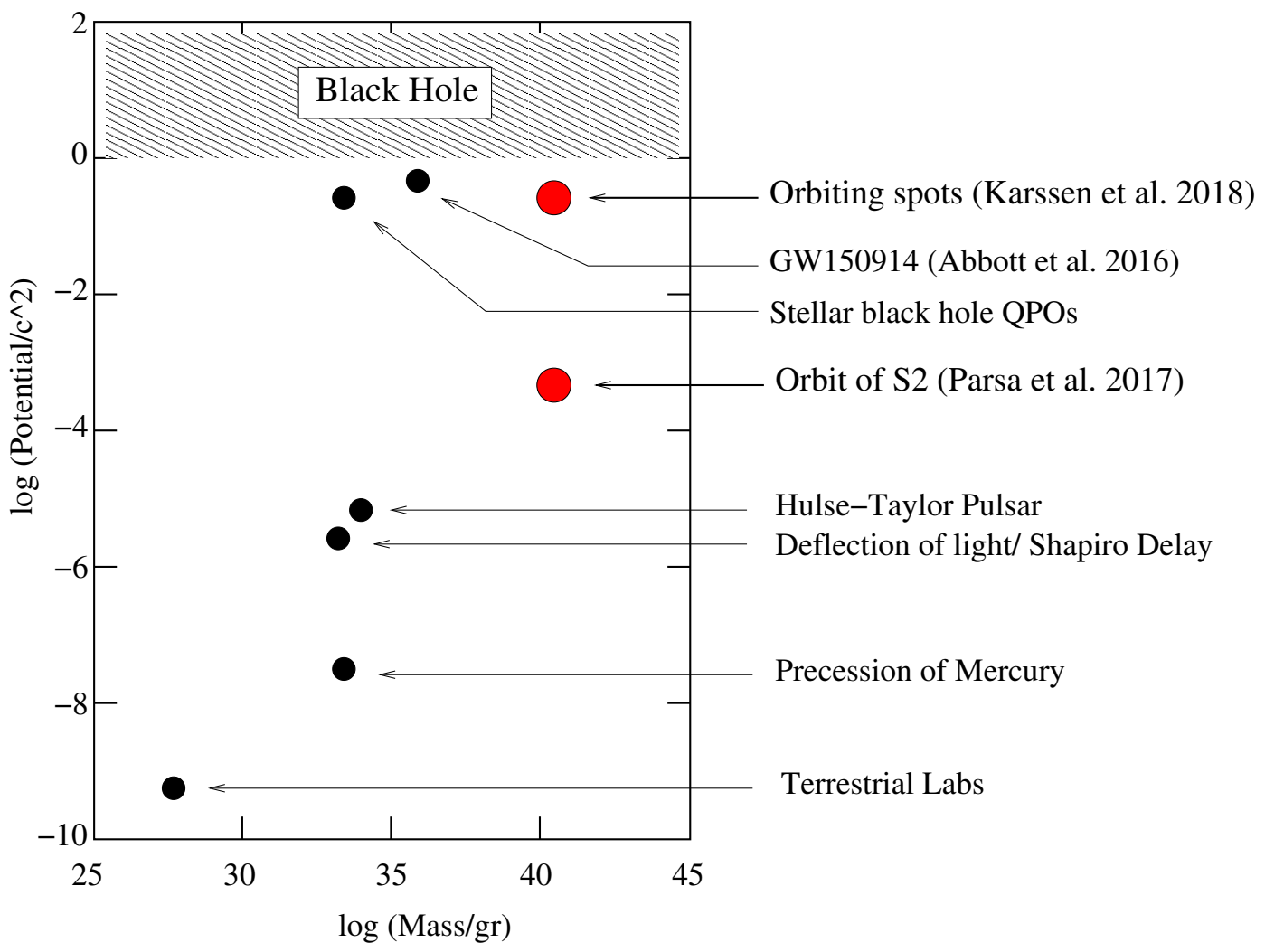

Figure 6: The gravitational potential that is measured by a number of tests of gravity presented in comparison to the mass responsible for the potential (based on Psaltis 2004). We have selected terrestrial labs, the precession of Mercury, light deflection and the Shapiro delay in the solar system, the Hulse-Taylor pulsar, QSO QPOs, and the gravitational wave detection GW150914 (Abbott et al. 2016). In addition we show the results for the Galactic Center S2 orbit presented by Parsa et al. (2017) and the X-ray lightcurve fitting presented by Karssen et al. (2017).

plasma. The CND appears to act as a blocking agent that keeps the hot plasma inside the central stellar cluster and close to the super massive black hole SgrA*.

The probes of the gravitational potential as they have been presented by Parsa et al. (2017) and Karssen et al. (2017) and summarized in the present publication can be compared to other tests in Fig. 6. This comparison is based on Fig.6 in Psaltis et al. (2004; see also Fig.1 in Hees et al. 2017, and Figs. 4 and 5 in Eckart et al. 2010). This figure shows that the gravitational potential can now be probed over 9 orders of magnitude for masses covering more than 10 orders of magnitude. These results can still be improved with currently running interferometry experiments in the radio mm-wavelength and infrared regime.

Acknowledgements: We received funding from the European Union Seventh Framework Program (FP7/2007-2013) under grant agreement No. 312789 - Strong gravity: Probing Strong Gravity by Black Holes Across the Range of Masses. This work was supported in part by the Deutsche Forschungsgemeinschaft (DFG) via the Cologne Bonn Graduate School (BCGS), the Max Planck 
Society through the International Max Planck Research School (IMPRS) for Astronomy and Astrophysics, as well as special funds through the University of Cologne and SFB 956 - Conditions and Impact of Star Formation. E. Hosseini is members of the IMPRS. We thank the Czech Science Foundation - DFG collaboration (No. 13-00070J) - and the German Academic Exchange Service (DAAD) for support under COPRAG2015 (No.57147386). The result on S2 has also been presented at the 'Stellar Dynamics in Galactic Nuclei' workshop, held at the Institute for Advanced Study, in Princeton, NJ, between November 29 and December 1, 2017, and at the 'Second Annual Black Hole Initiative Conference on Black Holes' (Harvard University), held at the Sheraton Commander Hotel, 16 Garden Street, Cambridge, MA, Wednesday, May 9 through Friday, May 11, 2018.

\section{References}

[1] Abbott, Benjamin P.; et al. (LIGO Scientific Collaboration and Virgo Collaboration) (2016). PhysRevLett.116.061102.

[2] Eckart, A., Schödel, R.; Garcia-Marin, M.; Witzel, G.; et al. 2008, A\&A 492, 337

[3] Eckart, A.; Zamaninasab, M.; Straubmeier, C.; et al., 2010, 2010SPIE.7734E..0XE

[4] Eckart, A.; Garcia-Marin, M.; Vogel, S. N.; Teuben, P.; et al., 2012, A\&A 537, 52

[5] Eckart, A.; Hüttemann, A.; Kiefer, C.; Britzen, S.; 2017, FoPh 47, 553

[6] Eckart, A., M. Zajacek, M. Parsa, et al. 2018, arXiv:1806.00284

[7] Event Horizon Telescope Collaboration; 2019a, ApJ 875, L1

[8] Event Horizon Telescope Collaboration; 2019b, ApJ 875, L5

[9] Falcke, H.; Melia, F.; Agol, E., 2000, ApJ 528, L13

[10] Gravity Collaboration 2017, A\&A 602, 94

[11] Gravity Collaboration 2018, A\&A 618, L10

[12] Hees, A.; Do, T.; Ghez, A. M.; Martinez, G. D.; et al., 2017, PhRvL.118u1101H

[13] Iorio, L., 2017, MNRAS 472, 2249

[14] Karssen G.D., Bursa M., Eckart A., Valencia-S M., et al., 2017, MNRAS 472, 4422

[15] Mossoux, E. \& Eckart, A., 2018, MNRAS 474, 3787

[16] Parsa, M.; Eckart, A.; Shahzamanian, B.; 2017, ApJ 845, 22

[17] Ponti, G.; George, E.; Scaringi, S.; Zhang, S.; et al.; 2017, MNRAS 468, 2447

[18] Psaltis, D., Wex, N. \& Kramer, M. 2016, ApJ 818, 121

[19] Martins, F., Gillessen, S., Eisenhauer, F., et al. 2008, ApJ 672, L119

[20] Mérand, A.; Berger, J.-P.; de Wit, W.-J.; Eisenhauer, F.; et al. 2017, The Messenger, vol. 170, p. 16-19

[21] Li, Y.-P. et al., 2015, ApJ, 810, 19

[22] Li, Y.-P.; Yuan, F.; Wang, Q.D.; 2017, MNRAS 468, 2552 
[23] Lu, Ru-Sen; Krichbaum, Thomas P.; Roy, Alan L.; et al., 2018, 2018arXiv180509223L, in press with ApJ

[24] Zajacek, M., \& Tursunov, A., 2018, contribution to the proceedings of the IBWS 2018 workshop (Carlsbad, https://www.ibws.cz/), 2018arXiv180411014Z 